\title{
Les révélateurs de l'identité chez Amélie Nothomb
}

\section{Résumé:}

Le discours litéraire d'Amélie Nothomb est singulier en ce qui concerne la question de l'identité. Sa création nous offre un terrain exceptionnel pour étudier l'influence du concept d'altérité et la place qu'il occupe dans le processus de la prise de conscience identitaire. Il est question de l'identité de Soi conçue comme un agrégat de désirs qui forme l'être, lui permet de se manifester et de se découvrir en faisant face à l'Autre qui représente l'altérité du monde qui l'entoure, qui stimule ses désirs et, d'une certaine manière, contribue à la prise de conscience de Soi et à la découvetre de l'identité. C'est la remise en cause de l'enracinement de l'être dans le monde qui l'entoure et la mise en valeur de son altérité.

Mots clés : Soi, identité, Autre, altérité, désir.

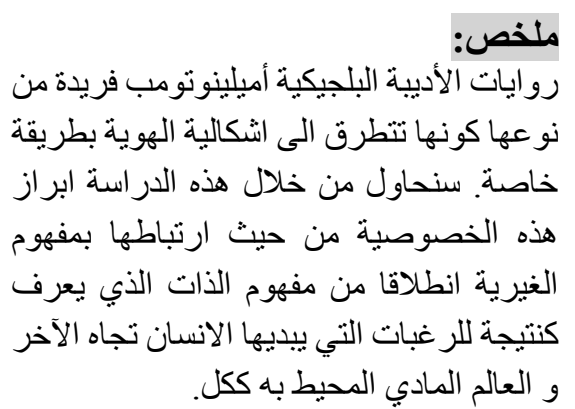

\section{Khadidja ATTIA}

Département des lettres et langue française Université des frères Mentouri Constantine 1

\begin{abstract}
Introduction :
Le discoursidentitaire est un discours en crise. Il s'inscrit dans l'ambivalence de ce qui est incertitude et construction. Il est dysharmonie, ambiguïté et reformulation. C'est pour cela qu'il ne fonctionne qu'en fonction des contextes.
\end{abstract}


La tâche que nous nous assignons consiste à montrer ce qu'il est convenu d'appeler « les révélateurs d'identité » chez l'écrivaine belge d'expression française Amélie Nothomb. C'est une tentative qui ne coïncide pas avec d'autres études qui ont essayé de répondre à la question de l'identité de l'être, traditionnellement résolue dans la recherche de ses origines ou de sa destinée en vue de savoir réellement qui il est. Ceci étant, il est important de cerner la résonance de ladite question chez cette romancière qui, par ses écrits, offre de nouvelles pistes dont la nature-même nous semble révélatrice d'une certaine complexité sur la question de l'être et de son identité.

Ce présent article entend aborder le thème de l'identité dans l'œuvre de Nothomb, en suivant la pensée du phénoménologue Emmanuel Lévinas. Ce dernier a inlassablement disséminé le concept d'identité dans presque tout son ouvrage Totalité et infini ${ }^{(1)}$. Cet ouvrage contient des réflexions et une indication que l'on peut essayer de suivre, non sans avoir préalablement expliqué en quoi consiste cette indication et quels sont les chemins qu'elle nous pousse à parcourir.

\section{1- Brèvesdéfinitions :}

- L'identité :

La notion d'identité constitue la question primordiale de la pensée humaine depuis le «Connais-toi toi-même » de Socrate jusqu'à ces jours, en passant par tant d'autres penseurs, philosophes et écrivains.

En fait, les plus grands auteurs affirment le lien étroit entre l'identité et l'existence. Selon Aristote, dans sa Métaphysique, un traité sur l'être et l'identité :

«Ces deux termes ne sauraient être séparés l'un de l'autre étant quasiment synonymes car l'identité est bien la relation que chaque individu (chose, entité) entretient avec lui-même tout au long de son existence ou de sa carrière. Dans son sens le plus fort, l'identité est une notion existentielle. $»^{(2)}$

Cependant, malgré les tentatives des chercheurs et spécialistes d'en donner une définition définitive, cette notion demeure encore ambiguë. Il serait donc difficile pour nous d'y proposer une définition concise et précise. De ce fait, nous faisons appel à de différents penseurs qui ont contribué, chacun sa façon, à l'explication et l'éclaircissement de ce concept.

Selon Ricœur, « identifier quelque chose, c'est pouvoir faire connaître à autrui, au sein d'une gamme de choses particulières du même type, celle dont nous avons l'intention de parler ${ }^{(3)}$

Pour sa part, Todorov définit l'identité comme « Le caractère de ce qui fait qu'un individu est lui-même et sedistingue de tout autre. ${ }^{(4)}$

Dans ces deux extraits, Ricœur et Todorov admettent que le concept de l'identité va de pair avec celui de l'altérité. Selon eux, c'est cette dernière qui permet au Moi de se définir par rapport à l'Autre. La présence de l'Autre estimpliquée dans 


\section{Les révélateurs de l'identité chez Amélie Nothomb}

ces deux définitions, illustrant fort bien la place qu'il occupe dans toute quête identitaire. L'altérité constitue la condition de l'émergence identitaire, que ce soit dans la pensée contemporaine ou dans celle de la philosophie antique. Les trois notions « altérité, identité, existence » sont inséparables l'une de l'autre : chaque existence cherche une identité, cette dernière se fait et se définit à travers des situations de mise en altérité.

\section{- Altérité et identité :}

Renvoyant essentiellement à une distinction entre le Même et l'Autre, le concept d'altérité a constitué, depuis les premières réflexions philosophiques, un couple avec la notion de l'identité. Etant donné que le propre se découvre par le différent, l'altérité peut permettre au Soi de se définir, d'affirmer son existence et de trouver son identité. De ce fait, la question de l'Autre c'est aussi une question du Soi et de son identité. La théorie lévinassienne de l'altérité dans Totalité et infini énonce que l'identification de Soi part de la relation concrète entre le Moi et le monde qui l'entoure, un Moi qui se définit par « le concret de l'égoisme. ${ }^{(5)}$ La relation qui les unit se produit comme dépendance de deux sortes d'éléments : des éléments qui s'offrent à la possession (matériaux désirés comme la nourriture) et des éléments qui se refusent à la possession (Autrui). Notre ambition se résume dans le but d'élucider le revirement de l'altérité du monde en identification de Soi.

\section{2- L'avènement identitaire chez Amélie Nothomb :}

Après des maintes lectures des œuvres nothombiennes, nous avons constaté que les personnages baignent dans des situations cataclysmiques relevant d'une certaine crise identitaire, régénérant des quêtes incessantes ayant pour but de se comprendre soi-même. Dès lors, se pose la question de l'identité de Soi.

Lévinas considère que le Soi représente un agrégat de désirs/ plaisirs qui forme l'être et lui permet de se manifester en tant qu'être dans tous les domaines de la vie humaine, d'où la conviction que la notion de désir est vitale dans le processus de la prise de conscience de Soi. L'analyse habituelle prétend qu'à la base du désir communément interprété, se trouverait le besoin. Selon Levinas, «Le désir marquerait un être indigent et incomplet ou déchu de sa grandeur du passé. Il coïnciderait avec la conscience de ce qui a été perdu. ${ }^{(6)}$

Le désir est donc un manque qui se produit en soi et que comble la dépendance inhérente au besoin, car parler de désir signifie parler de besoins. Et dans le but de comprendre ses enjeux, une réflexion sur le désir nous propulse non seulement dans sa profonde ambiguïté mais aussi dans celle de l'homme en son essence. Il est toujours désir de «quelque chose » qui manque à l'être. Ainsi, le désir pourrait se définir par la tension vers le désirable que celui-ci soit un 
objet, une personne ou encore un état de choses mettant l'être, d'emblée, face à une altérité du monde qui l'entoure. Il reste à montrer la fiabilité de la contribution de ce concept, en tant que source principale de la connaissance, dans le processus de la prise de conscience identitaire.

- L'être dans l'état de désir / plaisir :

"Au commencement, il n'y avait rien. " ${ }^{(7)}$ Dans Métaphysique des tubes, l'écrivaine raconte sa propre vie, de sa naissance jusqu'à l'âge de trois ans, marquée par l'expérience troublante du vide. Bébé, le monde extérieur lui est insignifiant. La petite Amélie évolue dans un univers entièrement clos qui l'empêche de construire une conscience, réduite à n'être qu'un corps.

Cependant, le moment où sa grand-mère paternelle lui offre une barre de chocolat blanc belge constitue une mise en scène fantasmatique ${ }^{(8)}$ qui permettra par la suite de dévoiler, de mettre en scène et traduire ses désirs inconscients. Lorsque le personnage principal subit un accident mental, ses parents invitent la grand-mère paternelle à venir rencontrer le bébé. Quand la grand-mère arrive de Bruxelles au Japon, dès le premier contact, elle approche de la bouche de l'enfant une barre de chocolat blanc belge et contribue à déclencher un processus de conscientisation :

« [...] La volupté lui monte à la tête, lui déchire le cerveau et y fait retentir une voix qu'il n'avait jamais entendue - c'est moi ! C'est moi qui vis ! [...] je ne suis pas "ill" ni "lui', je suis moi ! [...] le plaisir est une merveille qui m'apprend que je suis moi. ${ }^{(9)}$

C'est la grand-mère paternelle, donc, munie d'un bâton de chocolat qui entraîne chez l'enfant l'assimilation d'un Soi et l'avènement d'un Je dans le monde externe. Et c'est la jouissance alimentaire qui constitue le premier souvenir véritable : "En me donnant une identité, le chocolat blanc m'avait aussi fourni une mémoire. " ${ }^{(10)}$ La dégustation du bâton de chocolat constitue une expérience qui a permis l'avènement de la conscience de Soi chez le personnage principal, ainsi que l'avènement de la mémoire. Le plaisir, donc, l'a rendue opérationnelle, d'où le commencement de la quête identitaire par le biais du désir alimentaire.

A travers cette scène, la romancière déplace la prise de conscience identitaire du tout petit enfant, qui se découvre normalement dans le regard d'amour de sa mère, puis dans le miroir selon le psychanalyste Lacan $^{(11)}$, en le substituant par l'aliment sucré qui deviendra le déclencheur de plaisir chez elle. Alors, elle choisit de placer ce moment de découverte du chocolat belge dans le rang des évènements qu'elle considère à l'origine de son éveil par la découverte du plaisir. Il s'agit d'un thème très récurrent dans l'œuvre de Nothomb et qu'elle ne cesse de revendiquer de différentes manières à travers toutes ses œuvres car selon elle : 


\section{Les révélateurs de l'identité chez Amélie Nothomb}

« Le plaisir éveille l'esprit et le pousse tant à la virtuosité qu'à la profondeur. C'est une si puissante magie qu'à défaut de volupté, l'idée de volupté suffit. Du moment qu'existe cette notion, l'être est sauvé. » ${ }^{(12)}$

Le plaisir découvert donc est celui qui manifeste toute connaissance sensuelle et intellectuelle chez le personnage principal de Métaphysique des tubes et plus loin encore, il évolue et se mue en besoin qui, selon Lévinas, se trouve à la base du désir ${ }^{(13)}$. Ce désir se rencontrera dans d'autres romans de Nothomb, à travers des personnages comme celui d'Amélie dans le récit autobiographique Biographie de la faim, qui se découvre à son tour par le désir désormais conçu comme faim: «la faim, c'est vouloir. C'est un désir plus large que le désir "(14), notamment à travers la jouissance que lui assure «le sucre » qui lui donne l'occasion de découvrir la signification de son être :

«J'aurais aimé leur signifier qui j'étais, qui j'étais persuadée

d'être. J'étais le déferlement, l'être, l'absence radicale du non-

être $[\ldots]$, le dispenseur d'existence $[\ldots] \gg{ }^{(15)}$

Comme si, si l'on peut en déduire, tout être dépourvu du plaisir sera considéré et condamné tout simplement à «ne pas être ». La vie de Palamède Bernardin dans Les Catilinaires, est-elle ainsi considérée comme un "enfer ${ }^{(16)}$ puisqu'il n'éprouve aucun plaisir, ignorant même le "désir sexuel » ${ }^{(17)}$, d'où l'on peut considérer que les romans nothombiens ont pour ambition de doter le désir d'une influence considérable en matière de connaissance sensuelle.

La notion de plaisir, qu'engendre celle du désir, constitue dès lors l'élément déclencheur de toute prise de conscience identitaire chez les personnages principaux.

\section{- L'être dans la faim et le désir alimentaire :}

A côté de se découvrir par le plaisir, les personnages nothombiens se reconnaissent aussi dans la faim. Pour voir à quel point la notion de faim répond à celle du désir, nous allons revenir à cette notion dans différentes œuvres pour voir l'évolution de cette obsession. Nous allons établir une sorte de comparaison du statut de ce désir chez les personnages aux différentes phases de la vie : l'enfance, l'adolescence et l'âge adulte.

Une lecture attentive des œuvres nothombiennes ouvre sur le constat que les personnages se distinguent et se caractérisent par leur tentative incessante de trouver un principe explicatif à leur existence qui est celui de la faim. «Il s'agit d'abord des besoins du corps, mais ils éprouvent, par la suite, toutes sortes de faims : celle des aliments, la soif, la faim des Autres, des mots, etc., la faim de toutes possibilités de vie. » ${ }^{(18)}$ Une conduite qu'adoptent les personnages avec la conviction que la clef pour la découverte de Soi réside dans la faim : «Il s'agit de montrer que la faim est [la] plus haute identité. ${ }^{(19)}$ Ainsi Amélie, personnage principal de Biographie de la faim, a-t-elle cette intime conviction : 
« La faim, c'est moi [...] Par faim, j'entends ce manque effroyable de l'être entier, ce vide tenaillant, cette aspiration non pas tant à l'utopique plénitude qu'à la simple réalité : là où il n'y a rien, j'implore qu'il y ait quelque chose. »(20)

Il importe aussi de signaler que Biographie de la faim -qui suit les grandes étapes de la vie de la romancière - s'organise comme une démonstration virtuose de l'existence du personnage principal «Amélie » conçue comme faim, en retournant sur les moments de la belle faim enfantine qui déborde le sens commun et devient « surfaim » : surfaim du sucre, de l'eau et de l'alcool, qui ne tarde pas à se muer en besoin. Il reste à démontrer la relation qu'entreprend ce genre de besoin avec l'identité de l'être : Selon Lévinas, «la dépendance inhérente au besoin n'est pas aliénation mais bonnheur. ${ }^{(21)}$ Ce dernier engendre la jouissance que le personnage principal de Biographie de la faim pense comme la courbure même de sa " séparation » du monde qu'il habite et du " pour soi » de son être. Ainsi, le besoin est-il vécu comme signe du détachement qui institue l'identité. Sur ce point, la manière d'être selon laquelle est ou se vit un Moi qui consomme sa séparation, son indépendance et, ainsi, son identité en consommant le monde, confirme le rôle prépondérant que joue le plaisir, la faim, la complaisance au monde et à Soi dans le processus de la découverte de l'identité. Lévinas le confirme encore :

«Le paradoxe du "vivre de quelque chose" ou comme dirait Platon, "la folie de ces plaisirs", est précisément dans une complaisance à l'égard de ce dont la vie dépend. Non pas maîtrise d'une part et dépendance de l'autre, mais maîtrise dans cette dépendance. $\gg^{(22)}$

Se découvrir, pour le personnage principal de Biographie de la faim, c'est sentir la vie selon la saveur qui imprègne toutes les choses consommables de la vie et c'est ce qui fait la dignité de ce besoin, de cette faim : celle des nourritures, qui s'inscrit dans le corps comme son élévation, qui rend possible la transformation de la jouissance que lui procure ce plaisir en conscience identitaire.

Cependant, la découverte de Soi dans la faim des nourritures ne répond à la notion du désir qu'à l'enfance. Dès l'âge pubère, les personnages nothombiens « ne [vantent] plus les bénéfices de la belle faim enfantine, mais [craignent] un désir immodéré de se remplir au-delà de la faim. » ${ }^{(23)}$ Le corps pubère se révèle dès lors « obscène » s'il montre trop son goût du plaisir. Le désir alimentaire qui fait l'occasion d'une riche exploration pour le Soi dans l'enfance, se trouve moralisé dès l'âge pubère et devient objet de rejet et d'abandon, alors qu'il constituait, à l'enfance, l'objet d'une obsession. Cette contradiction (obsession/rejet) a pour origine « la nature du corps pubère qui, dans l'absorption démesurée de la nourriture, se révèle particulièrement et devient monstrueux dès qu'il ne peut plus être contrôlé. » ${ }^{(24)}$ 


\section{Les révélateurs de l'identité chez Amélie Nothomb}

Dans Biographie de la faim, dès l'âge de treize ans, Amélie «sombre dans la dictature de [son] corps. ${ }^{(25)}$ Elle subit une transformation qui constitue un signe de rupture avec l'enfance et, de ce fait, avec l'identité acquise à cette période. Pour surmonter cette privation "[elle] ferma [ses] frontières » ${ }^{(26)}$ par le biais de l'anorexie qui constitue dès lors le seul moyen de défense, la seule solution pour mettre fin à cette contradiction afin de restituer l'identité acquise à l'enfance. L'ancienne boulimie des plaisirs prend fin à l'adolescence avec l'anorexie dans laquelle le Soi acquis à l'enfance trouve, paradoxalement, la seule manière pour lui de survivre, en l'absence d'autres manières ou moyens de résistance ou de possibilités envisageables face à la tyrannie du corps pubère. Dans un tel cas de figures, on peut constater qu'il ne s'agit aucunement d'une conduite suicidaire qui naît chez les personnages mais plutôt, il s'agit d'une représentation du désir de résoudre le problème de la perte de l'identité acquise jadis par le biais du plaisir. Nous pouvons détecter la mise en scène de nombreux fantasmes propres à l'anorexie, notamment dans Hygiène de l'assassin où, pour maintenir son corps et celui de Léopoldine dans l'enfance, Prétextat Tach, le personnage principal de ce roman, a inventé une méthode particulièrement étrange :

«Vous inventez toutes sortes de mesures pseudo-scientifiques destinées à rendre vos corps impropres à l'adolescence [...] vous avez créé ex nihilo ce que vous appeliez une "hygiène de l'éternelle enfance"' - à l'époque, le domaine recouvrait tous les domaines de la santé mentale et physique : l'hygiène était une idéologie. Celle que vous inventez mériterait plutôt le nom d'anti-hygiène tant elle est malsaine [...] il ne faut plus dormir ou au moins pas plus de deux heures par jour. Une vie essentiellement aquatique vous paraît idéale pour retenir l'enfance $[\ldots] \gg{ }^{(27)}$

De cette manière, les deux adolescents ont réussi à garder leurs corps «immenses- maigres- blafards. " ${ }^{(28)}$ L'anorexie a Donc pour rôle de servir de moyen dans le processus de reconstruction chez les personnages qui se sentent « détruits » après avoir perdu leur identité acquise à l'enfance par le plaisir et, paradoxalement, l'anorexie la leur donne à la pubèrté. Mais, qu'en estil à l'âge adulte ?

A l'âge adulte, la nourriture redevient source de plaisir assurant la sensation identitaire que procurait la belle faim enfantine. Bien que le corps ne puisse plus être contrôlé car la stratégie de l'anorexie ne peut tenir jusqu'au bout. De ce fait, les personnages adultes sont le plus souvent « obèses ». Dans Les Catilinaires, le corps de Bernadette frappe par sa grande masse et se révèle « obscène » parce qu'il montre trop son goût du plaisir. Elle émet des grognements de contentement assimilés à un orgasme bestial lorsqu'elle éprouve du plaisir à se 
nourrir. Ce qui suscite chez Emile une forte répugnance : «Le spectacle de ce plaisir réjouissait et répugnait à la fois. ${ }^{(29)}$ Alors que dans Hygiène de l'assassin, Prétextat Tach signe volontairement une rupture avec l'âge idyllique suite à la mort de Léopoldine, et se transforme en un homme «laid et obèse » ${ }^{(30)}$ en devenant gourmand. Cependant, le plaisir alimentaire ne lui permet d'accéder qu'à des jouissances métissées de dégoût car il constitue une transgression de l'idéologie du corps impubère parfait à laquelle il croyait. Il n'est donc plus luimême, il est devenu un Autre que lui-même.

\section{- Le désirmétaphysiqued'Autrui :}

Aux abords de la pensée lévinassienne dans son ouvrage Totalité et infini, se trouve indiqué l'élément crucial dans la lutte pour l'identité de l'être : le combat quotidien contre les autres qui est remis en question par Amélie Nothomb à travers son écriture romanesque, en en faisant l'un de ses thèmes de prédilection.

L'Autre métaphysiquement désiré chez les personnages nothombiens n'est pas autre comme les autres choses dont ils ont besoin, dont ils ont faim et qu'ils peuvent satisfaire comme s'ils leur avaient simplement manqué, mais plutôt sa présence et son altérité aident à l'identification de Soi sans qu'il soit objet de satisfaction -contrairement au cas de la faim des nourritures- et dès lors :

«Ayant reconnu ses besoins comme besoins matériels, c'est-àdire comme capable de se satisfaire, le moi peut se tourner vers ce qui ne lui manque pas. Il [...] s'ouvre au désir ${ }^{(31)}$

Ce désir, dont parle Lévinas et auquel s'ouvre le Soi, est le désir d'Autrui qui émerge alors comme objet de besoin qui, étant différent du besoin matériel précédemment évoqué, constitue dès lors l'un des principaux mouvements du Même vers la connaissance de Soi.

Si le besoin du matériel peut être satisfait car pouvant tout simplement être absorbé par le Soi, le désir d'Autrui « entend l'absolument extérieur qui constitue même la signification de la notion de métaphysique. ${ }^{(32)}$ L'Autre métaphysique « est autre d'une altérité antérieure à toute initiative, à tout impérialisme du Même ${ }^{(33)}$ car le Même ne peut dominer l'Autre, sur lui, il ne peut rien, ils sont le Même et l'Autre, désireux et désiré qui sont totalement et absolument séparés l'un de l'autre. Dans ce cas, Lévinas conçoit que la relation métaphysique qui relie le Même à l'Autre consiste essentiellement « à transcender $»^{(34)}$ en partant de l'idée que « le transcendant est ce qui ne peut être englobé » ${ }^{(35)}$ ou absorbé. Le désir d'Autrui donc est un désir sans satisfaction qui, précisément, entend l'éloignement, l'altérité et l'extériorité de l'Autre. Pour le désir, cette altérité « [...] est entendue comme altérité d'Autrui et comme celle du Très-Haut. » ${ }^{(36)}$ C'est la définition même de la notion de transcendance qui exprime la distance qui existe entre le Même désireux et l'Autre désiré, et qui jalonne dès lors, toute relation entre les deux pôles de la rencontre à travers les œuvres nothombiennes. 


\section{Les révélateurs de l'identité chez Amélie Nothomb}

Puisque « la métaphysique aborde le désiré sans le toucher, sa manière n'est pas acte, mais relation sociale ${ }^{(37)}$, thème très présent dans l'œuvre romanesque nothombienne où la découverte de Soi se trouve liée très explicitement à la présence d'Autrui. La contribution directe de l'Autre à la découverte du Soi et de son identité est très récurrente chez les personnages d'Amélie Nothomb. Le sabotage amoureux en est la première illustration dont le personnage principal, Amélie l'enfant, qui se croyait le centre du monde en raison des temps paradisiaques qu'elle se procurait dans le Ghetto de San Li Tun, se trouve livrée à une quête identitaire des plus conflictuelles du moment où "[elle a] compris que le centre du monde, ce n'était pas [elle] » ${ }^{(38)}$, mais plutôt «le centre du monde se situait en dehors d'[elle] " ${ }^{(39)}$ et "il était de nationalité italienne et s'appelait Elena. " ${ }^{(40)} \mathrm{C}$ 'est donc le premier Autre que rencontre Amélie, dont elle s'est rendu compte, en a saisi la nature et le rôle qu'il joue ainsi que les effets qu'il produit sur elle. Alors Amélie « [faisait] tout pour $[s]$ 'en rapprocher ${ }^{(41)}$, quoi que Elena la rejette car non seulement elle est "Autre qui se refuse à la possession du Même, elle la conteste. » ${ }^{(42)}$ Le désir d'Autrui dans ce cas ne peut être satisfait car le désiré, au lieu de combler ce désir, le creuse davantage:

«J'aimais Elena et je sentais que cet amour appelait quelque chose. Je n'avais aucune idée de la nature de ce quelque chose [...] mais je sentais qu'après il devrait y avoir un échange obscur et indéfinissable. ${ }^{(43)}$

Malgrè les tentatives d'annuler l'écart qui sépare Amélie et Elena, cette dernière demeure transcendante et les tentatives de rapprochement que la petite Amélie fait pour pénétrer dans son lieu tournent à l'échec:

« [Elena] ne faisait jamais les premiers pas dans les contacts humains, parce qu'il fallait venir au devant d'elle pour entrer dans son monde, et parce qu'en fin de compte, personne n'entrait dans son monde. »(44)

Le désir métaphysique d'Autrui acquiert sa signification du moment où le personnage d'Amélie qualifie sa relation avec Elena, incarnation de l'Autre transcendant, de "surnaturel innomé ${ }^{(45)}$, expression qui figure pour qualifier l'échange obscur qui existe entre Elena et elle, cette Autre, froide et si peu curieuse vis-à-vis d'elle. Amélie se trouve, dans cet état de causes, incapable de révéler à l'Autre ce "quelque chose qui existe et qu' [elle] sent en [elle] et parce qu'il est mille fois plus beau que ce que [Elena] pourrait imaginer. " ${ }^{(46)}$ Mais, la rencontre avec Elena ne peut avoir pour résultat que ce qui est déjà présent mais dont Amélie n'est pas consciente. Ainsi, importe-t-il de souligner qu'en se retournant vers la conscience, vers le recherché qui est animé par un objectif visant autre chose que cette conscience (visant Autrui), et qui, toujours idée de quelque chose chez ce personnage, ouvre la voie de la 
conscience qui mène à découvrir où cette chose recherchée se loge et en quoi elle consiste. Les philosophes de l'existence admettent que le Même ne puisse rien recevoir d'Autrui, sinon ce qui est en lui, comme s'il possédait ce qu'il lui vient du dehors avant même la rencontre avec Autrui :

«Cette primauté du Même fut la leçon de Socrate, ne rien recevoir d'Autrui sinon ce qui est en moi, comme si, de toute éternité, je possédais ce qui me vient du dehors. » ${ }^{(47)}$

Cette possession n'est que le déploiement de l'identité dont Autrui joue désormais le rôle de révélateur permettant à ce « quelque chose » de trouver sa signification.

La deuxième œuvre qui constitue l'illustration par excellence du rôle d'Autrui dans la prise de conscience de Soi est l'œuvre intitulée Stupeur et tremblements, où l'affirmation de Soi est liée directement à la rencontre avec Autrui, dans le but de trouver un équilibre à une identité qui oscille entre japonisme et belgitude.

L'incipit de l'œuvre appelle le lecteur à avoir comme première impression le fait que le travail dans la compagnie Yumimoto suppose déjà la rencontre permanente avec le transcendant, en mettant l'accent sur son aspect hiérarchisé:

«Monsieur Haneda était le supérieur de monsieur Omochi, qui était le supérieur de monsieur Saito, qui était le supérieur de mademoiselle Mori, qui était ma supérieure. Et moi, je n'étais la supérieure de personne. $)^{(48)}$

En fait, pour l'ensemble du personnel dans la compagnie Yumimoto, l'appellation « Autre » renvoie à l'étranger. Dès son premier jour dans la compagnie, Amélie-San était consciente de sa place marginale dans cet endroit car le personnel de l'entreprise a tout fait pour lui faire comprendre son altérité. D'abord, elle est Autre parce qu'elle est une femme, "Geisha » ${ }^{(49)}$ faisant partie d'une minorité féminine. Ensuite, elle est Autre en raison de sa race, elle est «blanche ${ }^{(50)}$, elle n'appartient pas au groupe japonais. De plus, elle se trouve différente des autres en se mettant à l'écart du groupe à cause de son ignorance du savoir-vivre à la japonaise. Elle provoque le mécontentement de ses supérieurs, chose qui lui a causé le rejet de ces derniers, à l'exemple du malaise qu'elle a instauré lors de servir le café :

« Vous avez profondément indisposé la délégation de la firme amie [...] Monsieur Omochi est très fâché contre vous. Vous avez créé une ambiance exécrable dans la réunion de ce matin $[\ldots] \gg{ }^{(51)}$

Cependant, sa méconnaissance de ces codes a été, parfois, tolérée parce qu'- " elle est occidentale, elle est jeune, elle n'a aucune expérience » ${ }^{(52)}$ alors que, parfois, on lui reproche son incompétence, son infériorité par rapport aux Japonais : on lui fait comprendre qu'elle ne peut pas écrire une lettre ${ }^{(53)}$, qu'elle ne fait pas correctement des photocopies ${ }^{(54)}$ ce qui provoque, à chaque fois, le 


\section{Les révélateurs de l'identité chez Amélie Nothomb}

mécontentement de ses supérieurs. Sa situation s'aggrave davantage quand elle avoue sa rare stupidité face aux chiffres ${ }^{(55)}$ qu'elle considère comme des ennemis. Cette inaptitude accentue encore l'écart entre elle et les autres Japonais parce qu'au Japon, ce genre de personne n'existe pas ${ }^{(56)}$, à cause de quoi sa supérieure directe la traite d'idiote ${ }^{(57)}$, de handicapée mentale ${ }^{(58)}$, de même, elle l'accuse injustement de trahison et de sabotage. Ce genre d'humiliation verbale l'entraîne dans une dégradation d'ordre professionnel. Amélie-san, employée en qualité de traductrice, n'a pourtant fait que descendre, inexorablement, dans les degrés de la hiérarchie, pour devenir nettoyeuse des toilettes :

« Adulte, je ne résolus à être moins mégalomane et à travailler comme interprète dans une société japonaise. Hélas, c'était trop bien pour moi et je dus descendre un échelon pour devenir comptable. Mais, il n'y avait pas de frein à ma foudroyante chute sociale. Je fus donc mutée au poste de rien du tout. Malheureusement- j'aurais dû m'en douter- rien du tout, c'était encore trop bien pour moi. Et ce fut alors que je reçus mon affectation ultime : nettoyeuse des chiottes. ${ }^{(59)}$

Une telle humiliation à l'encontre de cette jeune occidentale, ne pourrait être que le fruit de plusieurs tentatives, de la part des Japonais, pour lui faire sentir son infériorité en tant qu'Occidentale et de s'en débarrasser. En effet, ils refusent la présence d'Amélie-san dans leur milieu, ils n'acceptent pas son Altérité.

Cependant, et malgré les rejets qu'elle subit, Amélie-San demeure dépendante de cette société d'accueil et ne cesse de chercher comment plaire à ses supérieurs sous prétexte de ne pas vouloir perdre la face ${ }^{(60)}$. Elle désire cet Autre qui la rejette, qui l'humilie. Peut être le conflit entre Amélie-san et sa supérieure directe n'est-il qu'une représentation simplifiée de cet univers de dualités extrêmes entre le Soi et l'Autre, si transcendant que le sujet ne peut le concevoir :

«- $[\ldots]$ je voulais que vous sachiez combien je suis déçue. Je vous tenais en si haute estime.

Elle eut un rire élégant :

- Moi, je ne suis pas déçue. Je n'avais pas d'estime pour vous. » ${ }^{(61)}$

La destinée d'Amélie alors lui a réservé d'être employée à Yumimoto, et son sort était d'y rester seule. Entre souhait et réalité, l'identité d'Amélie est remise en question en se balançant entre deux attitudes : la culture de son pays natal, qu'elle souhaitait s'y intégrer (le Japon), et celle de son pays d'origine (la Belgique). Il s'agit donc de la métaphysique d'Autrui doublée d'une problématique culturelle qui conduit à une problématique identitaire.

Dans LeSabotage Amoureux et Stupeur et tremblements, l'amour qu'éprouve Amélie pour Elena et Fubuki navigue entre un narcissisme persistant 
et un désir d'altérité. Amélie recherche l'amour chez l'une et l'amitié chez l'autre, dans une relation en miroir où l'Autre constitue une image d'être idéal qu'elle aimerait posséder.De plus, la rencontre avec Elena ou Fubuki lui permet de s'ouvrir à l'inconnu de l'Autre et à son étrangeté. C'est le danger d'une rencontre, de ce qu'elle engendre par la brisure qu'elle cause. Elena pour Amélie est la première Autre, elle n'a pas connu d'Autre jusqu'à présent. Elle voyait les gens autour d'elle mais elle n'avait pas besoin d'eux car elle ne se sentait pas dépendante d'eux, et tout à coup, il y a cet autre être dont l'existence est presque comme une menace puisque du coup l'enfant n'est plus autosuffisante.

A sept ans comme à vingt ans, Amélie ne sait pas encore marquer des limites à ne pas franchir. Elle donne tous les droits à l'Autre, celui de l'humilier, de la diminuer, sans pouvoir refuser la souffrance et se faire respecter.

Elena et Fubuki exploitent une faille effective chez Amélie qui, par son attitude, leur accorde une toute puissance dangereuse : nulle réciprocité entre Soi et l'Autre, l'un est le bourreau et l'autre victime.

Le désir de l'Autre n'est donc pas la négation de Soi. Il est l'aide à se mettre en question et l'appel à une quête identitaire. Cette mise en question ne consiste pas seulement à prendre conscience de son identité mais à aboutir à un être capable de recevoir une révélation, d'apprendre qu'il existe et de se mettre en question.

Cette présente partie, ayant pour but essentiel de mettre au clair les différents révélateurs d'identité chez l'écrivaine Amélie Nothomb, nous a permis d'entreprendre une analyse de ses textes pour mieux comprendre la visée de son écriture qui se veut un terrain de recherche pour des tentatives incessantes de se ressaisir soi-même. Nous avons démontré que le désir de l'Autre n'est aucunement la négation de Soi, mais plutôt une aide à se mettre en question et une invitation à une quête identitaire.

Cette manière de la prise de conscience de Soi, conçue dès lors comme seule et unique voie pour la révélation identitaire pour Amélie Nothomb, consiste à déborder les perspectives de la métaphysique humaniste, à prendre le risque de remettre en cause l'enracinement de l'être dans le monde qui l'entoure, ainsi que la mise en valeur de son altérité. Une voie par laquelle Nothomb, à la suite de Lévinas, est parvenue à dévoiler et à mettre en question le rapport entretenu par le Soi aux “choses", au corps, à la jouissance, à l'expérience, à la matérialité, à la faim. Ainsi, est-elle parvenue à tracer l'itinéraire qui permet le passage de l'ordre du Même à une trenscendance constituant Autrui. La relation avec l'altérité du monde qui entoure le Moi se produit comme désir. Parfois comme désir qu'apaise la possession du désirable, parfois comme désir jamais satisfait. Peut être, l'écrivaine Amélie Nothomb cherche, par le biais de son écriture, à attacher son nom à une doctrine un peu personnelle : la philosophie du désir, tout en essayant de convaincre son lecteur que le désir se trouve au cœur de la notion d'altérité. L'originalité de son écriture consisterait à cibler les expériences de la 


\section{Les révélateurs de l'identité chez Amélie Nothomb}

rencontre avec l'Autre, quelque soit sa nature, en tant que véritable altérité, révélation de l'Autre que l'on rencontre, tantôt en le possédant et en s'en réjouissant, tantôt sans pouvoir jamais ni le posséder, ni totalement le comprendre, ni le saisir, ni se l'approprier et s'en revendiquer, ouvrant la voie à d'autres questionnements.

\section{BIBLIOGRAPHIE :}

- AMANIEUX, Laureline. 2005. Amélie Nothomb : L'éternelle affamée. Albin Michel.

- FERRET, Stéphane. 1998. L'identité. Paris, Coll. « Corpus », GF Flammarion.

- LEVINAS, Emmanuel. [1961]. Totalité et infini : essai sur l'extériorité, Nijhoff, 1971.

- $\quad$ NOTHOMB, Amélie. 1992. Hygiène de l'assassin, Albin Michel.

- NOTHOMB, Amélie. 1993. Le sabotage amoureux, Albin Michel.

- NOTHOMB, Amélie. 1995. Les Catilinaires, Albin Michel.

- NOTHOMB, Amélie. 1999. Stupeur et tremblements, Albin Michel.

- NOTHOMB, Amélie. 2000. Métaphysique des tubes, Albin Michel.

- NOTHOMB, Amélie. 2004. Biographie de la faim, Albin Michel.

- $\quad$ RICOEUR, Paul. 1990. Soi-même comme un autre, Paris, Seuil.

- TODOROV, Tzvetan.1989. Nous et les autres. La réflexion française sur la diversité humaine, Paris, Seuil, Coll. « Points-Essais ».

\section{$\underline{\text { Notes : }}$}

(1)-Lévinas, Emmanuel. [1961]. Totalité et infini : essai sur l'extériorité, Nijhoff, 1971.

(2)- Cité par Ferret, Stéphane, L’identité, Paris, coll. « Corpus », GF Flammarion, 1998, p. 11

(3)- Ricœur, Paul, Soi-même comme un autre, Paris, seuil, 1990, p39

(4)- Todorov, Tzvetan, Nous et les autres. La réflexion française sur la diversité humaine, Paris, Seuil, Coll. « Points-Essais », 1989, p.33.

(5)- Lévinas, Emmanuel. Op cit, p8. 
(6)- Ibid., p22.

(7)- NOTHOMB, Amélie. Métaphysique des tubes, Albin Michel, 2000, p. 5

(8)-Amanieux, Laureline. Amélie Nothomb : L'éternelle affamée, Albin Michel, 2005, p21

(9)- NOTHOMB, Amélie. Op.cit., p31

(10)- NOTHOMB, Amélie. Op. Cit. p35.

(11)- AMANIEUX, Laureline. Op. Cit. p23

(12)- NOTHOMB, Amélie. Op. Cit. p34.

(13)-LEVINASs, Emmanuel. Op. Cit. p21

(14)- NOTHOMB, Amélie. Biographie de la faim, Albin Michel, 2004, p20.

(15)- NOTHOMB, Amélie. Biographie de la faim, Op. Cit. p31

(16)- NOTHOMB, Amélie. Les Catilinaires, Albin Michel, 1995, p133

(17)- Ibidem.

(18)- AMANIEUX, Laureline, Op. Cit. , p 258

(19)- NOTHOMB, Amélie. Biographie de la faim, Op. Cit. , p16

(20)- Ibid., p20

(21)- LEVINASs, Emmanuel. Op. Cit., p118.

(22)- Ibidem.

(23)- AMANIEUX, Laureline, Op. Cit. , p168.

(24)-Ibid., p167.

(25)- NOTHOMB, Amélie. Biographie de la faim, Op. Cit. , p162

(26)- Idem

(27)- NOTHOMB, Amélie..Hygiène de l'assassin, Albin Michel. 1992, p.p $110-111$.

(28)- Ibid., p121

(29)- NOTHOMB, Amélie. Les Catilinaires, Op. Cit. , p122.

(30)- NOTHOMB, Amélie. Hygiène de l'assassin, Op. Cit. , p147

(31)-LEVINAS, Emmanuel. Op. Cit. , p121

(32)-Ibid. , p111

(33)- LEVINAS, Emmanuel. Op. Cit. , p28

(34)- Ibid. , p293

(35)- Ibid. , p326

(36)- Ibid. , p23.

(37)- Ibid. , p111

(38)- NOTHOMB, Amélie. Le sabotage amoureux, Albin Michel. 1993, p31.

(39)-Ibid., p38.

(40)-Ibid., p32.

(41)-Ibid., p38.

(42)-LEVINAS, Emmanuel. Op. Cit. , p27

(43)-Ibid., p59.

(44)- NOTHOMB, Amélie. Le sabotage amoureux, Op. Cit. , p58.

(45)-Ibid., p60. 


\section{Les révélateurs de l'identité chez Amélie Nothomb}

(46)-Ibid., p62.

(47)-LEVINAS, Emmanuel. Op. Cit. , p34.

(48)- NOTHOMB, Amélie. Stupeur et tremblements, Albin Michel. 1999, p7.

(49)-Ibid., p27.

(50)-Ibid., p109.

(51)-Ibid., p20.

(52)-Ibid., p47.

(53)-Ibid, p11.

(54)-Ibid., p.p 33-34.

(55)-Ibid. p74.

(56)-Ibid. p68.

(57)-Ibid. p63.

(58)-Ibid. p68.

(59)-Ibid., p132.

(60)-Ibid., p22.

(61)-Ibid,, p57. 